this issue

\title{
Multiparametric Magnetic Resonance Imaging for Bladder Cancer: Development of VI-RADS (Vesical Imaging-Reporting And Data System)
}

\author{
Valeria Panebianco ${ }^{a, 1, *}$, Yoshifumi Narumi ${ }^{b, 1}$, Ersan Altun $^{c}$, Bernard H. Bochner ${ }^{d}$, \\ Jason A. Efstathiou ${ }^{e}$, Shaista Hafeez ${ }^{f, g}$, Robert Huddart ${ }^{f, g}$, Steve Kennish ${ }^{h}$, Seth Lerner ${ }^{i}$, \\ Rodolfo Montironi ${ }^{j}$, Valdair F. Muglia ${ }^{k}$, Georg Salomon ${ }^{l}$, Stephen Thomas ${ }^{m}$, \\ Hebert Alberto Vargas ${ }^{n}$, J. Alfred Witjes ${ }^{o}$, Mitsuru Takeuchi $^{p, 2}$, Jelle Barentsz $^{q, 2}$, \\ James W.F. Catto ${ }^{r, 2}$
}

\begin{abstract}
${ }^{a}$ Department of Radiological Sciences, Oncology and Pathology, Sapienza University of Rome, Italy; ${ }^{\mathrm{b}}$ Department of Radiology, Osaka Medical College, Takatsuki, Osaka, Japan; ' Department of Radiology, University of North Carolina at Chapel Hill, Chapel Hill, NC, USA; ${ }^{\mathrm{d}}$ Department of Surgery, Memorial Sloan-Kettering Cancer Center, New York, NY, USA; ${ }^{\mathrm{e}}$ Department of Radiation Oncology, Massachusetts General Hospital, Harvard Medical School, Boston, MA, USA; ${ }^{\mathrm{f}}$ The Institute of Cancer Research, Sutton, Surrey, UK; ${ }^{\mathrm{g}}$ The Royal Marsden NHS Foundation Trust, Sutton, Surrey, UK; ${ }^{\mathrm{h}}$ Department of Radiology, Sheffield Teaching Hospitals NHS Trust, Sheffield, UK; ${ }^{i}$ Scott Department of Urology, Baylor College of Medicine, Houston, TX, USA; ${ }^{\mathrm{j} S e c t i o n}$ of Pathological Anatomy, Polytechnic University of the Marche Region, School of Medicine, United Hospitals, Ancona, Italy; ${ }^{\mathrm{k}}$ Imaging Division, Ribeirao Preto Medical School, University of Sao Paulo, Ribeirao Preto, Brazil; ${ }^{1}$ Martini Clinic, University Clinic Hamburg Eppendorf, Hamburg, Germany; ${ }^{\mathrm{m}}$ Department of Radiology, University of Chicago, Chicago, IL, USA; ${ }^{\mathrm{n}}$ Department of Radiology, Memorial Sloan-Kettering Cancer Center, New York, NY, USA; ${ }^{\circ}$ Department of Urology, Radboud University Medical Center, Nijmegen, The Netherlands; ${ }^{\mathrm{p}}$ Department of Radiology, Radiolonet Tokai, Nagoya, Japan; ${ }^{\mathrm{q}}$ Department of Radiology, Radboud University Medical Center, Nijmegen, The Netherlands; ${ }^{\mathrm{r}}$ Academic Urology Unit, University of Sheffield, Sheffield, UK
\end{abstract}

\section{Article info}

Article history:

Accepted April 26, 2018

Associate Editor:

Giacomo Novara

Keywords:

Bladder cancer

Multiparametric magnetic

resonance imaging

Scoring

Staging

RADS

\begin{abstract}
Context: Management of bladder cancer (BC) is primarily driven by stage, grade, and biological potential. Knowledge of each is derived using clinical, histopathological, and radiological investigations. This multimodal approach reduces the risk of error from one particular test, but may present a staging dilemma when results conflict. Multiparametric magnetic resonance imaging (mpMRI) may improve patient care through imaging of the bladder with better resolution of the tissue planes than computed tomography and without radiation exposure.

Objective: To define a standardized approach to imaging and reporting mpMRI for BC, by developing a VI-RADS score. Evidence acquisition: We created VI-RADS (Vesical Imaging-Reporting And Data System) through consensus using existing literature.

Evidence synthesis: We describe standard imaging protocols and reporting criteria (including size, location, multiplicity, and morphology) for bladder mpMRI. We propose a five-point VI-RADS score, derived using T2weighted MRI, diffusion-weighted imaging, and dynamic contrast enhancement, which suggests the risks of muscle invasion. We include sample images used to understand VI-RADS.

Conclusions: We hope that VI-RADS will standardize reporting, facilitate comparisons between patients, and in future years, will be tested and refined if necessary. While we do not advocate mpMRI for all patients with BC, this imaging may compliment pathology or reduce radiation-based imaging. Bladder mpMRI may be most useful in patients with non-muscle-invasive cancers, in expediting radical treatment or for determining response to bladder-sparing approaches.

Patient summary: Magnetic resonance imaging (MRI) scans for bladder cancer are becoming more common and may provide accurate information that helps improve patient care. Here, we describe a standardized reporting criterion for bladder MRI. This should improve communication between doctors and allow better comparisons between patients.

(c) 2018 European Association of Urology. Published by Elsevier B.V. All rights reserved.
\end{abstract}

1 Joint lead authors.

2 Joint senior authors.

* Corresponding author. Department of Radiological Sciences, Oncology and Pathology, Sapienza University of Rome, Italy. Tel. +39 3358443792; Fax: +39 490243.

E-mail address: valeria.panebianco@uniroma1.it (V. Panebianco).

https://doi.org/10.1016/j.eururo.2018.04.029

0302-2838/@ 2018 European Association of Urology. Published by Elsevier B.V. All rights reserved. 


\section{Introduction}

\subsection{Bladder cancer}

Bladder cancer (BC) is one of the most common and expensive human malignancies to manage [1-3]. Most BCs are urothelial cell carcinomas (UCCs), and histologically stratified into cancers with low and high grade [4]. The latter are subdivided into those with and without muscle invasion. Non-muscle-invasive BCs (NMIBCs) are often low grade and have an indolent natural history [5]. Treatment is aimed at reducing local recurrence and stage progression, and maintaining quality of life [6]. High-grade lesions represent around one-third of NMIBCs, and can progress to muscle invasion and metastases in around $20-25 \%$ patients [7-9]. Treatment aims to reduce stage progression and preserve quality of life, while maintaining close surveillance to detect the onset of muscle invasion. Muscle-invasive BCs (MIBCs) are aggressive tumors with an ominous prognosis [10]. Success of treatment is dependent on the stage of the primary tumor and status of the regional lymph nodes. Cure can be achieved in $75-80 \%$ of patients with organ-confined disease, $60 \%$ of those with $\mathrm{T} 3$ node-negative disease and 30\% with lymph node-positive disease [11-15]. Despite increasing use of systemic therapy, overall survival rates from BC have not improved over the last $30 \mathrm{yr}$ [16] and these patients have some of the lowest healthcare experience ratings [17].

\subsection{Transurethral resection}

BC is usually detected by flexible cystoscopy following an episode of hematuria or for mixed urinary symptoms [6]. The diagnosis of $\mathrm{BC}$ is made by transurethral resection of bladder tumor (TURBT) of all or the most exophytic/ intraluminal tumor component. TURBT is used as definitive treatment for most NMIBCs and serves as a diagnostic procedure for most MIBCs. A properly performed TURBT samples the underlying bladder wall including the muscularis propria. Understaging occurs with TURBT, and it may miss muscle infiltration in up to $25 \%$ of invasive cancers [18-20]. TURBT is operator dependent, and so residual tumor rates (reflecting incomplete $\mathrm{BC}$ resection) vary widely with experience $[9,21]$. Re-resection of the bladder is advised in high-risk NMI cancers, prior to bladder preserving chemoradiation, or where the clinical and pathological findings differ $[6,22,23]$. Recent technological advances, such as narrow band imaging or the use of fluorescence agents ("blue light cystoscopy"), may improve the outcomes from TURBT [24,25]. While TURBT is the mainstay of diagnosis that has been used safely since the 1950s, there are concerns that it could lead to cancer embolization [26] and the oncological risks of perforation remain unknown [27].

\subsection{Radiological staging of $B C$}

The prognosis and management of $\mathrm{BC}$ mostly reflects tumor stage. For the primary tumor, this includes depth of invasion into the lamina propria, detrusor muscle, perivesical fat, adjacent organs, or pelvic side wall [28]. For metastases, this includes regional lymph nodes (number and location) and the presence of distant metastases. BC staging is accomplished through the combination of clinical (examination), pathological (TURBT specimens), and radiological means. Radiological examination should look for second urinary tract malignancies ( $5 \%$ of BCs may have an upper tract UCC) or other pathologies. Most guidelines suggest cross-sectional imaging for MIBCs and high-grade NMI cancers, due to the risks of invasion and regional or distant metastases, and upper urinary tract involvement.

\subsection{Potential role of mpMRI in BC care}

The multimodal approach to $\mathrm{BC}$ staging reduces the risk of error from one particular test, but may present a staging dilemma when results conflict [29,30]. Despite their apparent rigor, each modality is operator dependant and the concordance between individuals varies widely. For example, the quality of the TURBT will vary among surgeons, pathologists may disagree in BC grading (10-29\% discordance) and staging (15-56\% discordance) [4,31], while radiologists differ in agreement about muscle invasion [32]. Multi-parametric magnetic resonance imaging (mpMRI) offers an opportunity to reduce staging errors through better anatomical visualization [33,34]. Given its lack of radiation, mpMRI also offers a potentially safer tool to investigate individuals at risk of BC and to image the same patients prior to, during, and following treatment to determine response.

\section{Evidence acquisition}

\subsection{Materials and methods}

VI-RADS started with a nonsystematic literature review using Medline, PubMed, and Web of Science in July 2017. Search terms included "bladder cancer", "urothelial carcinoma”, "MRI," and "multi-parametric MRI." This fed organization of the subtopics and informed literature selection. The VI-RADS system was achieved through a Delphi-like consensus using multidisciplinary team members from Europe, North and South America, and Asia, in a combination of electronic and face-to-face rounds.

1. Panel members were asked to summarize the evidence in the given area and highlight areas of controversy. Members of the working group met in Chicago in November 2017 (RSNA meeting) to agree with the statements. A facilitator collated the proposals.

2. Members of the working group evaluated each proposal, based on evidence-based research and professional experience, before drafting VI-RADS and deriving consensus.

3. The manuscript was written by subteams for each topic, through a series of iterations before final voting by the entire working group on the final content. 
4. The most important controversies on the scoring system were solved through experience and prior literature.

\section{Evidence synthesis}

\subsection{Technical considerations for image acquisition}

\subsubsection{Clinical considerations}

3.1.1.1. Timing of MRI and bladder treatments. Previous TURBT and intravesical Bacille Calmette-Guerin (BCG) or chemotherapy instillation cause edema and inflammation in the bladder wall and surrounding perivesical tissues, which can be difficult to distinguish from BC (resulting in overestimation of local stage) [35]. As there is no reliable method to avoid or measure reactive change in the bladder wall, MRI examination is best performed before or at least 2 wk after TURBT, bladder biopsy, or intravesical treatment. Air in the bladder (from cystoscopy or indwelling catheter) can cause distortion of diffusion-weighted imaging (DWI) due to susceptibility artifact. A 2-3-d interval between cystoscopy or removal of Foley catheter and MRI examination is recommended if the patient's condition allows.

3.1.1.2. Patient preparation. Motion and susceptibility artifacts from bowel peristalsis can be minimized by the administration of an intramuscular antispasmodic agent [36]. Adequate bladder distension allows correct visualization of the wall and identification of the muscularis propria (detrusor) without any folds (Supplementary Fig. 1). Adequate bladder distention is vital and can be achieved by instructing the patient to void $1-2 \mathrm{~h}$ before imaging or by instructing the patient to start drinking $500-1000 \mathrm{ml}$ of water in the $30 \mathrm{~min}$ before the examination, depending on the patient's tolerance level [37,38]. Without distention, the bladder wall will appear thick and uneven, leading to either a misdiagnosis of $\mathrm{BC}$ or overstaging of tumors that are present. Overdistension of the bladder may cause a motion artifact due to discomfort, and the extent of $\mathrm{BC}$ will be indistinct. In patients with a history of incomplete bladder emptying, a residual volume ultrasound scan prior to MRI can be useful to judge when the bladder is optimally full (around $300 \mathrm{ml}$ ). Real-time MRI images can also be used to determine adequate bladder filling. For an underfilled bladder, the scan should be repeated in 30-60 min after the patient has drunk more fluid. In case of an overfilled bladder, the patient should partly empty their bladder before the scan is repeated.

\subsubsection{Technical considerations}

3.1.2.1. MR equipment and protocol optimization. MRI ( 1.5 or $3.0 \mathrm{~T})$ is recommended to achieve high spatial resolution and signal-to-noise ratio. A multichannel phased array external surface coil is also recommended.

3.1.2.2. Image acquisition. T2-weighted (T2W) imaging, DWI, and dynamic contrast-enhanced imaging (DCE MRI) are key components of mpMRI examination. All images should include the whole bladder, proximal urethra, pelvic nodes, and prostate if the patient is a male. In females, adjacent pelvic viscera (uterus, ovaries, fallopian tubes, and vagina) should also be included. Spin-echo T1-weighted (T1W) imaging is used to identify hemorrhage and clot in the bladder, and bone metastasis.

3.1.2.2.1. T2-weighted imaging. At least two planes of multiplanar (axial, coronal, and sagittal) T2W images without fat suppression are usually obtained with two-dimensional (2D) fast-spin-echo (FSE) or turbo-spin-echo sequences. Three-dimensional spin-echo acquisitions (eg, SPACE, CUBE, VISTA) may be used as an adjunct to $2 \mathrm{D}$ acquisitions. If acquired using isotropic voxels, an arbitrary plane perpendicular to tumor base can be reformatted. For 2D-FSE, slice thickness of 3-4 $\mathrm{mm}$ is recommended to maximize spatial resolution while maintaining the signal-to-noise ratio.

3.1.2.2.2. Diffusion-weighted imaging. DWI is computed by quantifying the diffusion of water molecules in tissues, and it plays a significant role in the bladder mpMRI examination. Axial and sagittal/coronal breathing-free spinecho EPI sequence combined with spectral fat saturation is recommended. A high $b$ value $\left(800-1000 \mathrm{~s} / \mathrm{mm}^{2}\right)$ is needed to visualize $\mathrm{BC}$ with high contrast to surrounding tissues; however, a too high $b$ value is unnecessary and it may degrade signal-to-noise ratio [39]. It is essential to obtain DWI with good image quality, maintaining a balance between high spatial resolution and signal-to-noise ratio. Consideration should be given to available tools and techniques to achieve this, including the use of parallel imaging with short echo time, increasing the number of excitations, and adjusting the matrix and corresponding voxel size (Table 1). A drawback of DWI is difficulty in understanding the anatomical location because of the signal suppression of the background. Therefore, locations should match or be similar to those used for T2W imaging to interpret DWI referencing T2W imaging [40].

3.1.2.2.3. Dynamic contrast enhanced imaging. Although either a $2 \mathrm{D}$ or a 3D $\mathrm{T} 1$ gradient echo (GRE) sequence with fat suppression may be used, 3D acquisition (eg VIBE, LAVA, THRIVE) is preferred to obtain higher spatial resolution [41]. Precontrast imaging is also acquired. A gadoliniumbased contrast agent is administered using a power-injector system at a dose of $0.1 \mathrm{mmol} / \mathrm{kg}$ of body weight at a rate of $1.5-2.0 \mathrm{ml} / \mathrm{s}$ if standard relaxivity agent is used and followed by saline flush [42]. Initial contrast image is acquired (midline of k-space is filled) at $30 \mathrm{~s}$ after the beginning of injection and followed by the same sequences four to six times every $30 \mathrm{~s}$ to depict the early enhancement of inner layer followed by tumor enhancement [43]. If 3DGRE is acquired with isotropic voxels, an arbitrary plane perpendicular to tumor base can be reformatted. Late phase is not useful for T staging because signal contrast among the inner and outer layers and tumor decreases.

3.1.2.2.4. Semiquantitative/quantitative measurement (optional). Several studies are exploring quantitative measurements, such as apparent diffusion coefficient (ADC) and perfusion curves, to find objective markers for MRI tumor evaluation. 
Table 1 - Examples of parameter setting (1.5 and $3.0 \mathrm{~T})$

\begin{tabular}{|c|c|c|c|}
\hline & $\mathrm{T} 2 \mathrm{~W}$ & DWI & DCE MRI \\
\hline \multicolumn{4}{|l|}{ Parameter setting at $1.5 \mathrm{~T}$} \\
\hline TR (ms) & 5000 & 4500 & 3.3 \\
\hline $\mathrm{TE}(\mathrm{ms})$ & 80 & 88 & 1.2 \\
\hline Flip angle (degree) & 90 & 90 & 13 \\
\hline FOV $(\mathrm{cm})$ & 23 & 27 & 35 \\
\hline Matrix & $256 \times 189-256$ & $128 \times 109$ & $256 \times 214$ \\
\hline Slice thickness (mm) & 4 & 4 & 2 \\
\hline Slice gap (mm) & $0-0.4$ & $0-0.4$ & 0 \\
\hline Number of excitations & $1-2$ & $10-15$ & 1 \\
\hline$b$ values & & $0-800-1000$ & \\
\hline \multicolumn{4}{|l|}{ Parameter setting at $3.0 \mathrm{~T}$} \\
\hline TR (ms) & 4690 & 2500 up to 5300 & 3.8 \\
\hline $\mathrm{TE}(\mathrm{ms})$ & 119 & 61 & 1.2 \\
\hline Flip angle (degree) & 90 & 90 & 15 \\
\hline $\mathrm{FOV}(\mathrm{cm})$ & 23 & 32 & 27 \\
\hline Matrix & $400 \times 256-320$ & $128 \times 128$ & $192 \times 192$ \\
\hline Slice thickness (mm) & $3-4$ & $3-4$ & 1 \\
\hline Slice gap $(\mathrm{mm})$ & $0-0.4$ & $0.3-0.4$ & 0 \\
\hline Number of excitations & $2-3$ & $4-10$ & 1 \\
\hline$B$ values & & $0-800-1000$ (up to) $2000 \mathrm{~s} / \mathrm{mm}^{2}$ & \\
\hline
\end{tabular}

$\mathrm{DCE}=$ dynamic contrast enhancement; $\mathrm{DWI}=$ diffusion-weighted imaging; FOV = field of view; $\mathrm{MRI}=$ magnetic resonance imaging; $\mathrm{TE}=\mathrm{echo}$ time; $\mathrm{TR}=$ repetition time; $\mathrm{T} 2 \mathrm{~W}=\mathrm{T} 2$ weighted.

Wash-in and washout rates may be used as semiquantitative parameters. Three regions of interest may be considered, placed respectively on the intravesical tumor, at the bladder wall immediately below the lesion (detrusor/tumor interface) between the bladder mucosa and detrusor muscle, and on a normal detrusor muscle remote from the lesion. In low-grade tumors, the contrast washout is higher than for high-grade cancers [44]. On perfusion weighted imaging, the k-trans allows the evaluation of capillary permeability, which is indirectly an expression of tumor neoangiogenesis [45]. Greater permeability was present in the intraluminal tumor and in the detrusor wall for muscle-invasive cancers [46].

Several authors have reported that ADC values differ between low, intermediate, and high histological grade tumors, suggesting a possible correlation between imaging and histopathology. A lack of conclusive ADC cutoff values and different techniques applied (different $b$ values) limits this semiquantitative tool. Diffusion tensor imaging has been reported, and fractional anisotropy is an objective index that increases at the interface between the tumor and the bladder wall in MIBC [46].

3.1.2.2.5. Example of parameter setting. Although the optimal scan parameter depends on MRI scanner, examples of representative parameters are shown in Table 1.

\subsection{Scoring and reporting of images}

\subsubsection{Rationale}

In VI-RADS, we aim to standardize bladder mpMRI for clinical and research applications. In particular, we try to create a systematic approach to reporting bladder MRI and defining the risk of muscle invasion (NMIBC vs MIBC). The scoring is applicable to untreated patients (uVI-RADS scoring) and to patients having received "only" diagnostic TURBT (tVI-RADS), before re-TURBT.

\subsubsection{Anatomy}

3.2.2.1. Histology. The bladder wall includes three basic layers: mucosa, muscularis propria (detrusor muscle), and perivesical fat. The mucosa includes surface urothelium and underlying subepithelial connective tissue (or lamina propria). Normal urothelium is seven or fewer cells thick, hyperplastic and dysplastic lesions can be thicker, and in carcinoma in situ cells lose adhesion. The subepithelial connective tissue depth is similar for the anterior, posterior, and lateral walls $(0.72-2.55 \mathrm{~mm})$, but thinner at the trigone (ie, $0.46-1.58 \mathrm{~mm}$ ) and thicker at the dome $(0.98-3.07 \mathrm{~mm})$ [47]. The subepithelial connective tissue contains a muscularis mucosae. This consists of wavy, thin, smooth muscle fascicles often associated with large, thin-walled blood vessels. It can be identified in 15-83\% of biopsy specimens. Discernable muscularis mucosae is missing in 6\% radical cystectomy specimens. In such situation, the large vessels can be used as a surrogate marker of muscularis mucosae [47-50]. The connective tissue between the muscularis mucosae and the muscularis propria is occasionally called "submucosa" by radiologists. The muscularis propria is composed of inner and outer smooth muscles with different orientations. The boundary between the muscularis propria and perivesical tissue is not well defined. Aggregates of adipose tissue are often seen in muscularis propria, and in $61 \%$ are abundant in deep muscularis propria. The muscularis propria adipose tissue merges with perivesical adipose tissue without a clear line of demarcation from the perivesical tissue [48]. Tumorrelated factors such as dense fibrosis, inflammation, and tumor cells at the edge of outermost muscularis propria can make the distinction between muscularis propria and perivesical tissue difficult [48]. 
3.2.2.2. MRI appearances of anatomy. MRI does not have the necessary spatial resolution to visualize all the histological bladder wall layers (Fig. 1) [51]. Muscularis propria (detrusor) appears as a low signal intensity (SI) line on T2W images, while the inner layer composed of urothelium and lamina propria is not seen. At DWI, the inner layer is not visualized, while muscularis propria appears as an intermediate SI line. With ADC maps, urine appears hyperintense and bladder wall is of intermediate SI. With DCE, the inner layer presents early enhancement, and it appears as a high SI line, while muscularis propria presents as a low SI line that enhances slowly and progressively [42,51]. Clinicians should be aware that several conditions can cause inflammation of urothelium and lamina propria, resulting in thickening and edema. In such cases, T2W images may show a thickened hyperintense line (ie, the edematous inner layer) that overlays the hypointense muscular layer [51]. DWI may show a thickened hypointense line representing edematous mucosa.

3.2.2.3. MRI appearances with benign bladder pathologies. The bladder wall structure and MRI appearance may mimic cancer in various benign diseases and with some treatments (see section 3.2 for more detail). It is therefore important to correlate MRI findings with the clinical history, treatments received, and cystoscopic appearances. For example, cystitis cystica and cystitis glandularis produce flat and exophytic growth patterns, with low SI on T1W and T2W images, and hypervascular stalks with intact muscularis propria [47]. Inflammatory myofibroblastic tumors (IMFs), characterized by proliferation of plump, stellate, or elongated spindle cells with inflammatory cells and edematous or myxoid stroma containing a delicate network of small blood vessels, may also be misdiagnosed as cancer. On T2W MR images, IMFs appear heterogeneous, with a central hyperintense component surrounded by a low SI periphery; after administration of contrast material, the periphery enhances while the central region enhances poorly [47]. With regard to treatments, in the long term, TURBT causes fibrosis and chronic inflammation, replacing the normal bladder wall components and often leading to thickening of the wall. Chronic inflammatory or fibrotic tissues have lower cellular density than cancer, so that restriction is not so evident. Nevertheless, as they can show early enhancement at DCE MRI, DWI and ADC are crucial for differential diagnosis [49,50]. BCG therapy can lead to bacterial and immune-mediated cystitis, granulomatous inflammation, and bladder contracture, each of which may mimic a recurrent tumor [48]. Intravesical chemotherapy may produce similar changes. Radiotherapy to the pelvis (and bladder) in the short term may cause hemorrhagic cystitis, intraluminal clots, edema, and inflammation of the bladder. On MRI, these appear as focal or diffuse irregular thickening, within a bladder with decreased distensibility (low bladder volume) and hypervascularity. MRI may reveal high SI of the inflamed and edematous bladder wall at T2W sequences [47]. In the long term, radiation may result in interstitial fibrosis, with low SI on T2W images [47].

\subsubsection{Recording Bladder lesions}

3.2.3.1. MRI definition of the lesion. Intravesical lesions with $\mathrm{T} 2$ SI intermediate to urine and muscle, a high DWI signal and a low signal at ADC map, and postcontrast early enhancement at DCE MRI (Fig. 1) should be recorded as suspicious lesions.

3.2.3.2. Mapping. A schematic map is recommended to record the tumor location (example in Fig. 2). About one-third of new tumors arise from the trigone, bladder neck, and ureteral orifice regions, and a slightly greater number from the lateral walls [31]. Bladder neck cancers have a significantly higher frequency of muscle invasion [52]. The presence of multiple tumors should be recorded,

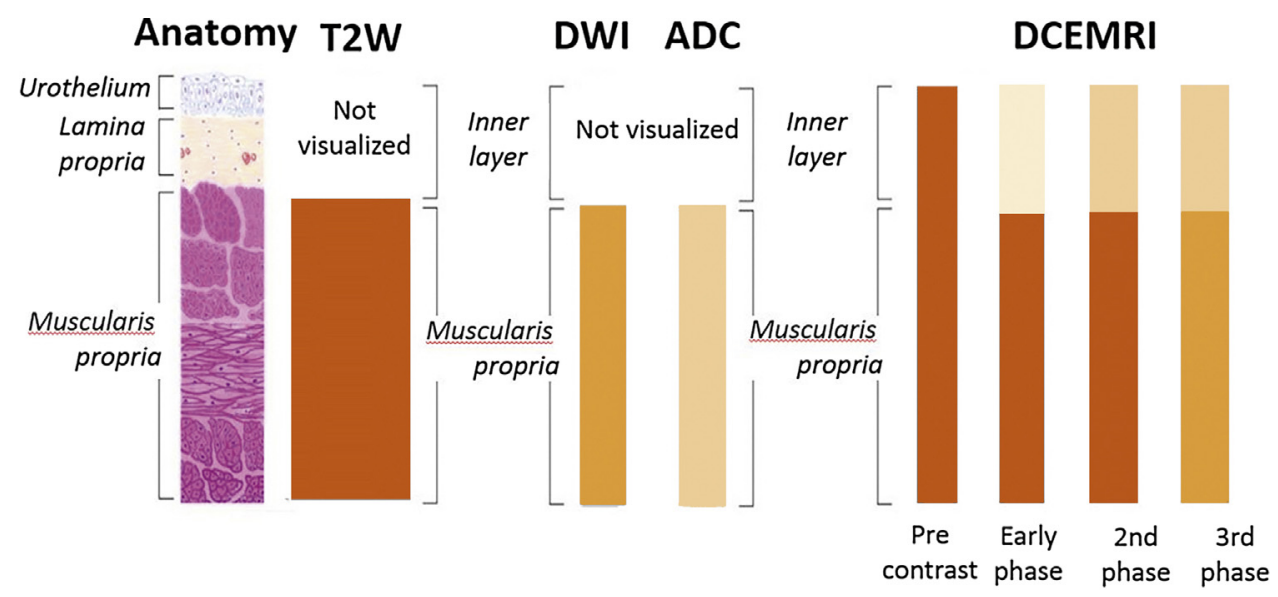

Fig. 1 - Schematic appearance of bladder wall anatomy and respective MRI appearances at T2W imaging, DWI, ADC, and DCE MRI. T2W images show low SI of muscular layer, and cannot visualize/discriminate the urothelium and the lamina propria. At DWI, the muscular layer appears as an intermediate SI line, while inner layer is not visualized; ADC maps shows intermediate signal of muscular layer compared with high signal of urine. The bladder wall components change appearance during the phases of DCE imaging. ADC = apparent diffusion coefficient; DCE = dynamic contrast enhancement; DWI = diffusion-weighted imaging; $\mathrm{MRI}$ = magnetic resonance imaging; $\mathrm{SI}$ = signal intensity; $\mathrm{T} 2 \mathrm{~W}=\mathrm{T} 2 \mathrm{weighted}$. 


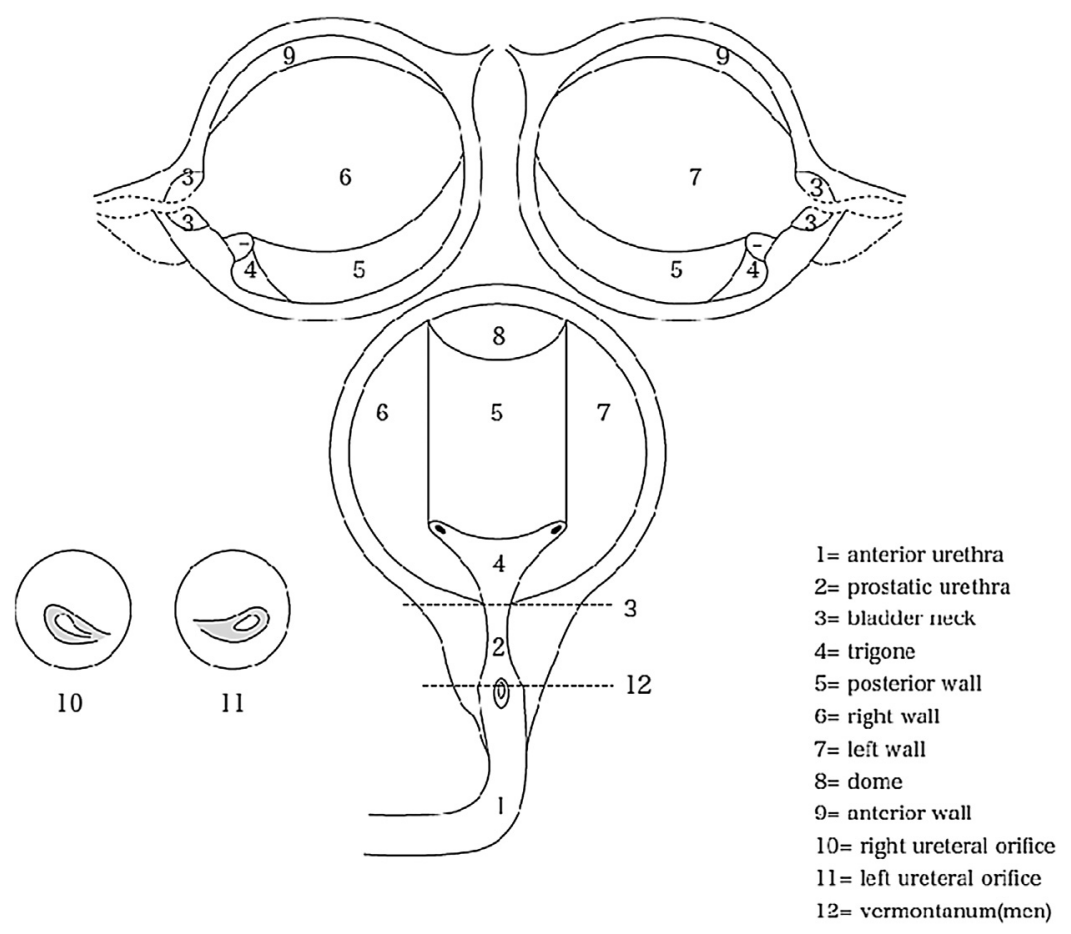

Fig. 2 - Schematic map of the bladder modified from the Japanese Urological Association, the Japanese Society of Pathology, Japan Radiological Society schema (published in General rule for clinical and pathological studies on renal pelvic, ureteral and bladder cancer, Kanehara Publisher Inc., 2011, p. 16).

together with details of the greatest disease burden (largest or highest number) and the tumor with radiological appearance of most advanced stage.

3.2.3.3. Morphology (size, growth pattern, stalk). Tumors may be of endophytic (intramural growth), exophytic (endoluminar growth), flat (nonmass effect), and mixed forms. The exophytic form can be papillary broad based or pedunculated; papillary tumors with a stalk are generally of better prognosis that papillary tumors without a stalk or broad sessile cancers. Stage T1 tumors with stalks (median diameter $21.5 \mathrm{~mm}$ ) are generally larger than those without stalks (13 mm) at T1 stage [49,53]. A tumor average size of 21.8, 23.2, and 33.7 was found in low-, intermediate-, and high-grade BC, respectively [44]. Small and flat BC ( $<1 \mathrm{~cm}$ of diameter) may have no stalk, and have the same T2 SI and enhancement pattern of the inner bladder layer. While this can cause an overestimation, tumor burden using DCE imaging [46], DWI, and T2W imaging help improve accuracy.

\subsection{4. $\quad$ MRI scoring}

3.2.4.1. Scoring. We propose scoring tumors using appearances in T2W imaging, DWI, and DCE to create an overall risk of invasion score (Figs. 3 and 4, and Supplementary Fig. 2). Normal bladder mucosa/inner layer shows high T2W SI and early phase enhancement in DCE MRI. Normal bladder mucosa/inner layer is not seen on T2W and DWI sequences under normal circumstances; however, these may be seen as a high SI structure on T2W images and a low SI structure on DWI if seen due to edema or inflammation. Normal muscularis propria shows low SI on T2W images and no early enhancement at DCE MRI. Visualization of different muscular layers is difficult to achieve, but can be seen as low T2 SI of the innermost muscular layer (muscularis propria) and intermediate intensity in the outer part [51].

3.2.4.2. Assessment for $T 2 W$ imaging. Muscle appears hypointense on T2W images. This should be the first MR appearance to search in the examination. Interruption of the low SI muscular line may suggest muscle invasion. Different scenarios may be present $[48,49,53]$ :

1. Structural category (SC) 1: uninterrupted low SI line representing the integrity of muscularis propria (lesion $<1 \mathrm{~cm}$; exophytic tumor with or without stalk and/or thickened inner layer)

2. SC 2: uninterrupted low SI line representing the integrity of muscularis propria (lesion $>1 \mathrm{~cm}$; exophytic tumor with stalk and/or high SI thickened inner layer, when present, or sessile/broad-based tumor with high SI thickened inner layer, when present)

3. SC 3: lack of category 2 findings with associated presence of an exophytic tumor without stalk, or sessile/broad-based tumor without high SI thickened inner layer but with no clear disruption of low SI muscularis propria

4. SC 4: interruption of low SI line suggesting extension of the intermediate SI tumor tissue to muscularis propria 


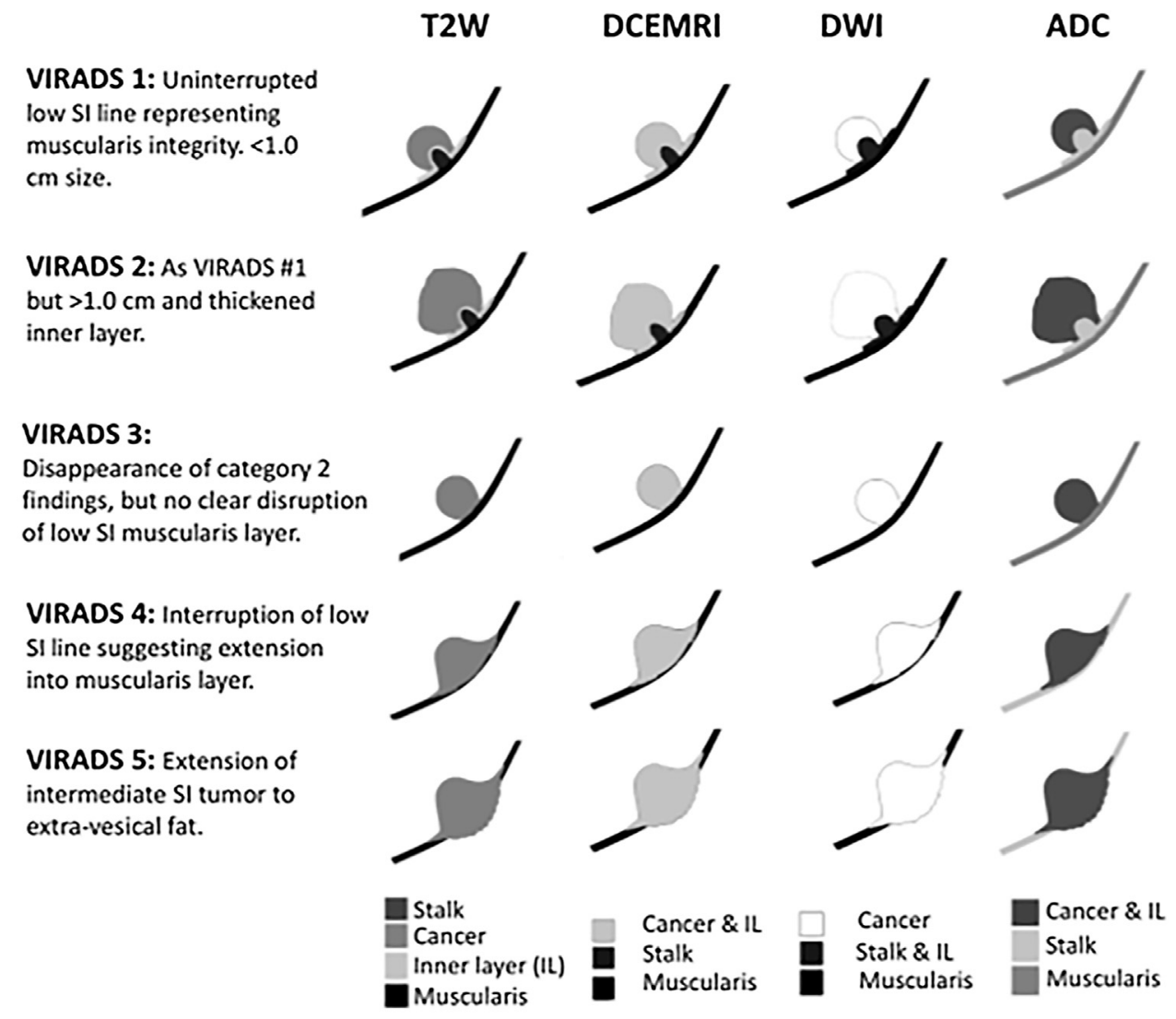

Fig. 3 - Schematic illustration of mpMRI appearances of VI-RADS scores 1-5 using T2, DCE MRI, DWI, and ADC. ADC = apparent diffusion coefficient; DCE = dynamic contrast enhancement; DWI = diffusion-weighted imaging; MRI = magnetic resonance imaging; mpMRI = multiparametric MRI; SI = signal intensity.

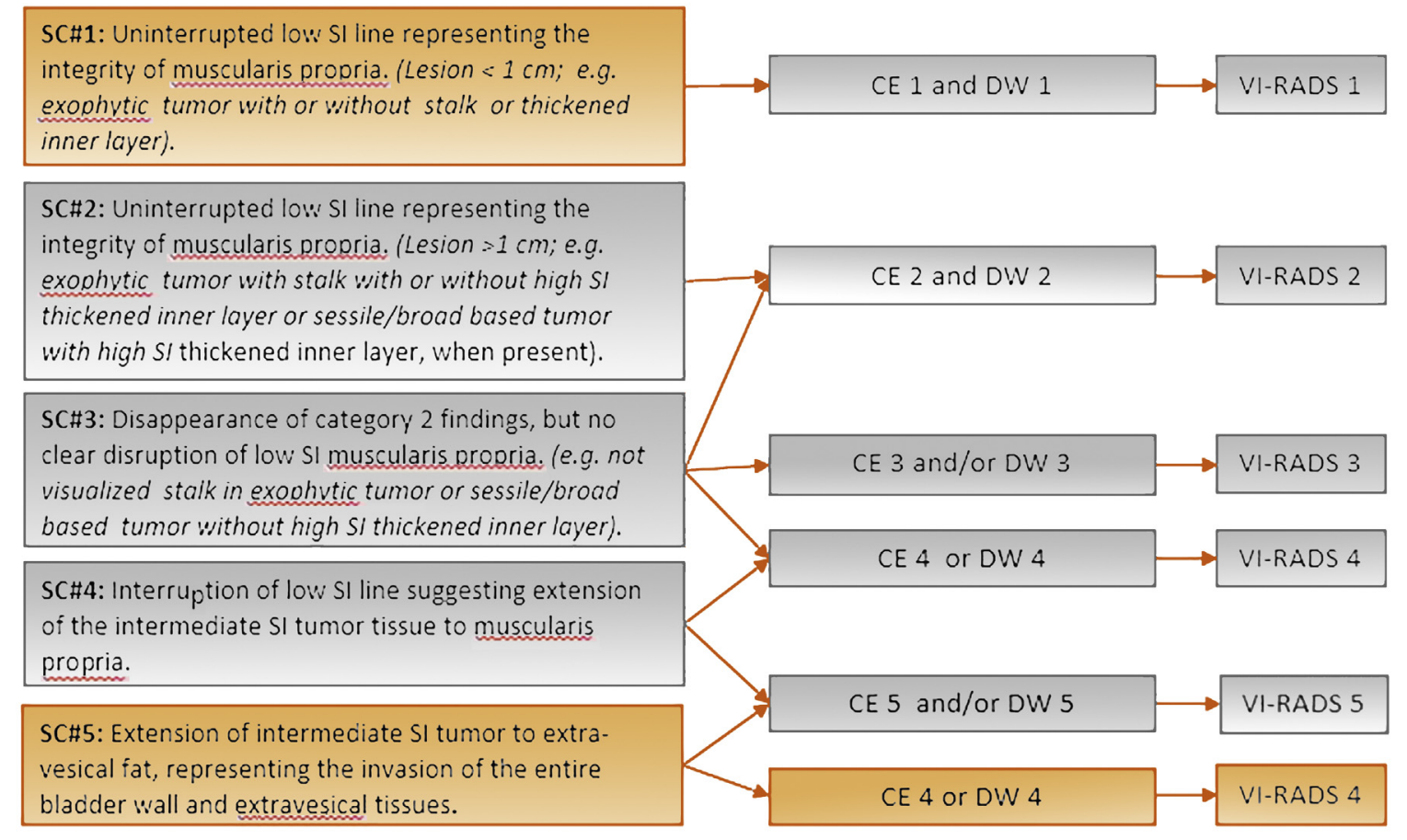

Fig. 4 - Summary schematic representation of VI-RADS scoring. Scoring interpretation: for categories 1-3, the "first pass scoring" T2 sequence should be considered. For categories 4 and 5 , the dominant sequences are DWI (first, when image quality is optimal) and DCE (second). CE = contrastenhanced category; DCE = dynamic contrast enhancement; $D W=$ diffusion-weighted category; DWI = diffusion-weighted imaging; SC = structural category; SI = signal intensity. 
5. SC 5: extension of intermediate SI tumor to extravesical fat, representing the invasion of the entire bladder wall and extravesical tissues

3.2.4.3. Assessment for DCE MRI. As for DCE MRI, tumor and inner layer enhance early and may enhance at the same time and grade. Muscularis propria should maintain no enhancement in the early phase, and it is recognizable as a low SI line under the tumor $[44,46,48,51]$. Contrast-enhanced (CE) categories are as follows:

1. CE category 1: No early enhancement of the muscularis propria (lesions corresponding to SC 1 findings)

2. CE category 2: no early enhancement of muscularis propria with early enhancement of inner layer (lesions corresponding to SC 2 findings)

3. CE category 3: lack of category 2 findings (lesions corresponding to SC category 3 findings) but with no clear disruption of low SI muscularis propria

4. CE category 4: tumor early enhancement extends focally to muscularis propria

5. CE category 5: tumor early enhancement extends to the entire bladder wall and to extravesical fat

3.2.4.4. Assessment for $D W I / A D C$. The tumor is hyperintense on DWI and hypointense on ADC map. Muscularis propria may present intermediate SI, while the stalk and inner layer have low SI on DWI [51,53]:

1. DW category 1: muscularis propria with intermediate continuous SI on DWI (lesion $<1 \mathrm{~cm}$, hyperintense on DWI and hypointense on ADC, with or without stalk and/ or low SI thickened inner layer on DWI)

2. DW category 2: muscularis propria with continuous intermediate SI on DWI (lesion $>1 \mathrm{~cm}$, hyperintense on DWI and hypointense on ADC, with low SI stalk and/or low SI thickened inner layer on DWI, or broad-based/ sessile tumor with low/intermediate SI thickened inner layer on DWI)

3. DW category 3: lack of category 2 findings (lesions corresponding to T2 category 3 findings) but with no clear disruption of low SI muscularis propria.

4. DW category 4: High SI tumor on DWI and low SI tumor on ADC extending focally to muscularis propria.

5. DW category 5: High SI tumor on DWI and low SI tumor on ADC extending to the entire bladder wall and extravesical fat.

\subsubsection{Final scoring}

A five-point VI-RADS score is generated using the individual T2W, DWI, and DCE MRI categories and suggests the probability of muscle invasion (Fig. 4, Supplementary Fig. 2, and Table 2). The dominant sequences for risk estimates are DWI (first) and DCE (second; especially if the DWI is suboptimal). The T2 sequence is helpful as a first pass guide, especially for categories $1-3$ :

1. VI-RADS 1 (muscle invasion is highly unlikely): SC, CE, and DW category 1
Table 2 - Example of VI-RADS report template

\begin{tabular}{l} 
Clinical history: \\
Clinical indications: \\
MRI acquisition protocol: \\
Findings: \\
Mapping lesion/s: Index lesion \\
Morphology: \\
Measurements of the lesions: \\
Scoring: \\
T2W \\
DWI \\
DCE \\
Final VI-RADS assessment (categories): \\
Semi/quantitative analysis (optional) \\
Transmural extension: \\
Adjacent organ invasion: \\
Nodes ${ }^{\text {a }}$ : \\
Bone: \\
Comments: \\
\hline DCE = dynamic contrast enhancement; DWI = diffusion-weighted imaging; \\
MRI = magnetic resonance imaging; T2W = T2 weighted. \\
a Criteria for pathological pelvic lymph nodes: (1) short axis 7.0 mm; (2) \\
round morphology; ( 3 ) loss of internal architecture; and (4) nodal \\
extracapsular extension.
\end{tabular}

2. VI-RADS 2 (muscle invasion is unlikely to be present): SC, $\mathrm{CE}$, and DW category 2; both CE and DW category 2 with SC category 3

3. VI-RADS 3 (the presence of muscle invasion is equivocal): SC, CE, and DW category 3; SC category 3, CE or DW category 3 , the remaining sequence category 2

4. VI-RADS 4 (muscle invasion is likely): at least SC and/or DW and CE category 4; the remaining category 3 or 4; SC category 3 plus DW and/or CE category 4; SC category 5 plus DW and/or CE category 4

5. VI-RADS 5 (invasion of muscle and beyond the bladder is very likely): at least SC plus DW and/or CE category 5; the remaining category 4 or 5

The final score is firstly based on T2W imaging for the morphology, because of their high spatial resolution in the evaluation of the integrity of muscularis propria. The presence of definitive muscular invasion is decided by DWI and DCE MRI. If there is any discordance between T2W and DCE sequences (a deviation of two categories between T2W and DCE MRI), DWI improves the accuracy $[33,38,40,46]$ when the image quality of DWI sequence is optimal.

\subsection{Discussion: clinical Impact}

\subsubsection{Overview}

MRI may be most promising in the pre-TURBT setting, given that the tissue architecture has not been distorted by surgery (ie, similar to preprostate biopsy). The current standard for diagnosis and staging BC is TURBT [6]. Cystoscopy has improved with enhanced intravesical imaging, such as photodynamic diagnosis and narrow band imaging, and better awareness of its importance in BC care [24]. However, many tumors are understaged by TURBT or detrusor is missing, and so complimentary improvements in imaging are 
welcomed [54,55]. An image-guided approach could identify and simultaneously stage a bladder tumor, such that a flexible cystoscopy might be skipped and patients be taken directly to TURBT. While histological confirmation remains the cornerstone of BC diagnosis [28], this pathway might be faster than reliance on TURBT [56]. For MRI to be a significant factor in clinical care, it has to be reproducible and feasible in the general hospital setting.

\subsection{2. mPMRI and NMIBC}

For NMI tumors, it is important to know the stage (pTa, pTIs, and pT1) to help choose treatment. Of note, MRI is less reliable at CIS detection. Of NMI cancers, accurate staging is most vital for a T1 tumor. MRI could help inform tumor size and likely stage, and help plan treatment. For example, for a small $\mathrm{T} 1$ tumor with a clear stalk and no muscularis propria involvement, a radical TURBT should be the goal and intravesical BCG therapy could be initiated without the need for a re-TURBT. For a deeper invading $\mathrm{T} 1 \mathrm{~T} 1 \mathrm{~b} / \mathrm{c})$ tumor, with no clear stalk or growth close to the muscularis propria, a re-TUR should be mandatory and thought is given to primary cystectomy [57,58]. Perhaps in the future, MRI with anatomical and functional information could predict tumors unlikely to respond to conservative therapy, for which the primary approach should be more radical [59]. Finally, knowing that approximately $25 \%$ of T1 tumors are muscle invasive on re-TURBT, which obviously changes therapeutic approach completely, accurate staging in T1 tumors might very well be one of the most important fields for MRI in BC staging $[19,60]$.

\subsection{3. $\quad$ mPMRI and MIBC}

For invasive, nonmetastatic BCs, there are different therapeutic strategies that can depend on the exact stage of the tumor. For example, stage could be used to guide the relative benefits from neoadjuvant chemotherapy [61]. For bladder-sparing therapy and chemoradiation, MRI could identify likely suitable tumors (unifocal, good bladder capacity) and help plan a radical, complete TURBT [62].

\subsection{4. mPMRI and treatment response}

Although cystoscopy remains the standard means of posttreatment follow-up, the need for frequent repeat examinations has driven mpMRI applications as a noninvasive alternative [63-65]. Data suggest qualitative and quantitative DWI, and DCE MRI change has correlation to pathological and clinical outcomes. This has potential important use in predicting treatment sensitivity and monitoring therapeutic response, particularly in patients receiving neoadjuvant chemotherapy or radical chemoradiotherapy for muscle-invasive disease.

Post-treatment inflammation and fibrosis cause irregular bladder wall thickening. This produces high SI on T2W imaging, which makes it challenging to distinguish between BC and benign treatment-related effects [15,66-68]. As a result, the reported sensitivity, specificity, and accuracy of $\mathrm{T} 2 \mathrm{~W}$ imaging to predict complete pathological response following chemoradiotherapy are low $(43 \%, 45 \%$, and $44 \%$, respectively) [67]. Treatment-related effects can persist for up to $18-24 \mathrm{mo}$, so the timing of $\mathrm{T} 2 \mathrm{~W}$ imaging acquisition is largely ineffectual in aiding judicious clinical decision making [68].

DCE MRI appears to successfully overcome the limitations of distinguishing between treatment-related effects and residual tumor following TURBT [69]. However, Wang et al [50] demonstrated that DWI is superior to DCE MRI in identifying tumor recurrence following either TURBT or partial cystectomy. The reported accuracy, sensitivity, specificity, and positive predictive values of DWI are $93 \%$, $100 \%, 82 \%$, and $89 \%$, respectively, compared with $59 \%, 81 \%$, $27 \%$, and $54 \%$, respectively, for DCE MRI.

Complete response following induction treatment is associated with improved survival and informs candidate selection for radical bladder preservation protocols [7076]. Widely investigated induction strategy employs TURBT and neoadjuvant chemotherapy and chemoradiotherapy to 40 Gy with complete responders as identified on cystoscopy and biopsy proceeding to a consolidative course of chemoradiotherapy to a total dose of $64-65$ Gy $[73,75]$. Another approach is assessing complete response following fulldose chemoradiation to 64-60 Gy.

The feasibility of mpMRI to assess therapeutic response to induction therapy was demonstrated by Yoshida et al [67]. mpMRI performed after induction chemoradiotherapy for T2-T4a NOM0 BC showed that DWI was significantly superior in specificity (92\%) and accuracy (80\%) to both T2W (45\% and $44 \%$ ) and DCE MRI (18\% and 33\%) in predicting pathological response. All sequences however showed poor sensitivity for detecting residual disease (43-57\%).

Pretreatment DWI predicts sensitivity to induction chemoradiotherapy. Treatment-sensitive bladder tumors have significantly lower ADC values than chemoresistant tumors (median $0.63 \times 10^{-3} \mathrm{~mm}^{2} / \mathrm{s}$ vs $0.84 \times 10^{-3} \mathrm{~mm}^{2} / \mathrm{s}$; $p=0.001$ ) [77]. Considering other clinical variables such as age, $T$ stage, tumor size, tumor multifocality, presence of carcinoma in situ, and hydronephrosis, only mean ADC was identified as an independent predictor of chemoradiotherapy sensitivity on multivariate analysis [77]. One explanation for this is that tumor ADC correlates with cell cycle regulators and molecular proliferative markers including p53, p21, and Ki-67 [77-79].

The survival benefit of neoadjuvant chemotherapy is recognized for those with MIBC [61,80,81]. On DCE MRI, significant change from early to later enhancement following three cycles of neoadjuvant chemotherapy is associated with complete pathological response at cystectomy [41]. Persisting early enhancement is indicative of poor chemotherapy response [41,63]. Combining semiquantitative DCE MRI parameters improves differentiation between residual bladder tumor and chemotherapy related effects with reported sensitivity and specificity of $75 \%$ and $100 \%$, respectively [41].

Neoadjuvant chemotherapy is not without toxicity. Patients who do not respond are exposed to morbidity and a delay in effective treatment [74]. Early mpMRI scanning may offer opportunity to inform treatment selection, with early response supporting continued use or poor response supporting treatment switch. Nguyen et al [65] used K-means cluster maps of DCE MRI to show that 
microvascular changes seen midcycle can be used to differentiate between responders and nonresponders to neoadjuvant chemotherapy.

In patients with advanced tumors, who may receive up to six cycles, prediction of overall response to chemotherapy can be made with DCE MRI after two cycles with greater sensitivity, specificity, and accuracy than conventional MRI $[63,64]$.

DWI has a role in assessing neoadjuvant chemotherapy response. A significant increase in ADC from baseline is seen in those achieving complete pathological response compared with incomplete responders and is associated with improved overall survival and time to cystectomy [82,83]. Example of a responder and nonresponder as seen on DWI after three cycles of chemotherapy is shown in Supplementary Fig. 3. DWI also provides predictive information of neoadjuvant chemotherapy response, as resistant bladder tumors appear to be more heterogeneous in their spatial distribution of ADC [84].

Multiparametric MRI appears a promising tool to monitor treatment response. However, the few currently available reports either are small in size or have design limitations. As such, mpMRI is not ready for standard care. Current challenges include standardization of data acquisition and analysis, validation, and prospective testing within clinical trials [85].

\section{Conclusions}

Multiparametric MRI may improve the care of men with prostate cancer through facilitating better diagnosis (by targeting of the biopsy, avoiding biopsy, or identifying missed cancers) and better treatment (by planning and stratifying risk). The creation and updating of the Prostate Imaging Reporting and Data System gave the foundations for these improvements [86]. Here, we hope to have created a foundation for mpMRI in BC staging, diagnosis, and therapeutic response, through a consensus-driven approach to standardized imaging and reporting. We hope that VI-RADS is user friendly, will allow simplified reporting, and in future years will be tested, validated, and refined where necessary. While we do not advocate mpMRI for all patients with $\mathrm{BC}$, this imaging may compliment pathology or reduce radiation-based imaging.

\section{Endorsement of VI-RADS}

VI-RADS has been endorsed as a reporting system for the bladder by the Japanese Society of Abdominal Radiology, European Association of Urology, and European Society of Urological imaging.

Author contributions: Valeria Panebianco had full access to all the data in the study and takes responsibility for the integrity of the data and the accuracy of the data analysis.

Study concept and design: Panebianco, Narumi, Catto. Acquisition of data: Takeuchi, Muglia, Vargas.
Analysis and interpretation of data: Altun, Takeuchi, Muglia, Lerner, Kennish, Thomas.

Drafting of the manuscript: Panebianco, Catto, Takeuchi, Narumi, Montironi, Hafeez.

Critical revision of the manuscript for important intellectual content: Montironi, Witjes, Bochner, Efstathiou, Salomon, Huddart, Barentsz.

Statistical analysis: None.

Obtaining funding: None.

Administrative, technical, or material support: Panebianco, Takeuchi, Narumi, Hafeez.

Supervision: Catto.

Other: None.

Financial disclosures: Valeria Panebianco certifies that all conflicts of interest, including specific financial interests and relationships and affiliations relevant to the subject matter or materials discussed in the manuscript (eg, employment/affiliation, grants or funding, consultancies, honoraria, stock ownership or options, expert testimony, royalties, or patents filed, received, or pending), are the following: None.

Funding/Support and role of the sponsor: None.

Acknowledgments: The authors thank Isabella Ceravolo from the Department of Radiological Sciences, Oncology and Pathology, Sapienza University of Rome, Italy.

\section{Appendix A. Supplementary data}

Supplementary data associated with this article can be found, in the online version, at https://doi.org/10.1016/j. eururo.2018.04.029.

\section{References}

[1] Svatek RS, Hollenbeck BK, Holmang S, et al. The economics of bladder cancer: costs and considerations of caring for this disease. Eur Urol 2014;66:253-62.

[2] Antoni S, Ferlay J, Soerjomataram I, Znaor A, Jemal A, Bray F. Bladder cancer incidence and mortality: a global overview and recent trends. Eur Urol 2017;71:96-108.

[3] Leow JJ, Cole AP, Seisen T, et al. Variations in the costs of radical cystectomy for bladder cancer in the USA. Eur Urol 2018;73:374-82.

[4] Soukup V, Capoun O, Cohen D, et al. Prognostic performance and reproducibility of the 1973 and 2004/2016 World Health Organization grading classification systems in non-muscle-invasive bladder cancer: a European Association of Urology Non-muscle Invasive Bladder Cancer Guidelines Panel systematic review. Eur Urol 2017;72:801-13.

[5] Linton KD, Rosario DJ, Thomas F, et al. Disease specific mortality in patients with low risk bladder cancer and the impact of cystoscopic surveillance. J Urol 2013;189:828-33.

[6] Babjuk M, Bohle A, Burger M, et al. EAU guidelines on non-muscleinvasive urothelial carcinoma of the bladder: update 2016. Eur Urol 2017;71:447-61.

[7] Sylvester RJ, van der Meijden AP, Oosterlinck W, et al. Predicting recurrence and progression in individual patients with stage Ta T1 bladder cancer using EORTC risk tables: a combined analysis of 2596 patients from seven EORTC trials. Eur Urol 2006;49:466-75, discussion 75-7.

[8] Kluth LA, Black PC, Bochner BH, et al. Prognostic and prediction tools in bladder cancer: a comprehensive review of the literature. Eur Urol 2015;68:238-53.

[9] Gontero P, Sylvester R, Pisano F, et al. Prognostic factors and risk groups in T1G3 non-muscle-invasive bladder cancer patients initially 
treated with Bacillus Calmette-Guerin: results of a retrospective multicenter study of 2451 patients. Eur Urol 2015;67:74-82.

[10] Witjes JA, Comperat E, Cowan NC, et al. EAU guidelines on muscleinvasive and metastatic bladder cancer: summary of the 2013 guidelines. Eur Urol 2014;65:778-92.

[11] Fonteyne V, Ost P, Bellmunt J, et al. Curative treatment for muscle invasive bladder cancer in elderly patients: a systematic review. Eur Urol 2018;73:40-50.

[12] Noon AP, Albertsen PC, Thomas F, Rosario DJ, Catto J. Competing mortality in patients diagnosed with bladder cancer: Evidence of undertreatment in the elderly and females. Br J Cancer 2013;108:1534-40.

[13] Stein JP, Lieskovsky G, Cote R, et al. Radical cystectomy in the treatment of invasive bladder cancer: long-term results in 1,054 patients. J Clin Oncol 2001;19:666-75.

[14] James ND, Hussain SA, Hall E, et al. Radiotherapy with or without chemotherapy in muscle-invasive bladder cancer. N Engl J Med 2012;366:1477-88.

[15] Giacalone NJ, Shipley WU, Clayman RH, et al. Long-term outcomes after bladder-preserving tri-modality therapy for patients with muscle-invasive bladder cancer: an updated analysis of the Massachusetts General Hospital experience. Eur Urol 2017;71:952-60.

[16] Eylert MF, Hounsome LS, Persad R, et al. Falling bladder cancer incidence from 1990 to 2009 is not producing universal mortality improvements. J Clin Urol 2014;7:90-8.

[17] Saunders CL, Abel GA, Lyratzopoulos G. Inequalities in reported cancer patient experience by socio-demographic characteristic and cancer site: evidence from respondents to the English Cancer Patient Experience Survey. Eur J Cancer Care (Engl) 2015;24:85-98.

[18] Kulkarni GS, Hakenberg OW, Gschwend JE, et al. An updated critical analysis of the treatment strategy for newly diagnosed high-grade T1 (previously T1G3) bladder cancer. Eur Urol 2010;57:60-70.

[19] Gordon PC, Thomas F, Noon AP, Rosario DJ, Catto JWF. Long-term outcomes from re-resection for high-risk non-muscle invasive bladder cancer: a potential to rationalize use. Eur Urol Focus. In press. https://doi.org/10.1016/j.euf.2017.10.004.

[20] Ark JT, Keegan KA, Barocas DA, et al. Incidence and predictors of understaging in patients with clinical T1 urothelial carcinoma undergoing radical cystectomy. BJU Int 2014;113:894-9.

[21] Brausi M, Collette L, Kurth K, et al. Variability in the recurrence rate at first follow-up cystoscopy after TUR in stage Ta T1 transitional cell carcinoma of the bladder: a combined analysis of seven EORTC studies. Eur Urol 2002;41:523-31.

[22] Hall MC, Chang SS, Dalbagni G, et al. Guideline for the management of nonmuscle invasive bladder cancer (stages Ta, T1, and Tis): 2007 update. J Urol 2007;178:2314-30.

[23] Burger M, Oosterlinck W, Konety B, et al. ICUD-EAU international consultation on bladder cancer 2012: non-muscle-invasive urothelial carcinoma of the bladder. Eur Urol 2013;63:36-44.

[24] Witjes JA, Babjuk M, Gontero P, et al. Clinical and cost effectiveness of hexaminolevulinate-guided blue-light cystoscopy: evidence review and updated expert recommendations. Eur Urol 2014;66:863-71.

[25] Mari A, Abufaraj M, Gust KM, Shariat SF. Novel endoscopic visualization techniques for bladder cancer detection: a review of the contemporary literature. Curr Opin Urol 2018;28:214-8.

[26] Engilbertsson H, Aaltonen KE, Bjornsson S, et al. Transurethral bladder tumor resection can cause seeding of cancer cells into the bloodstream. J Urol 2015;193:53-7.

[27] Mydlo JH, Weinstein R, Shah S, Solliday M, Macchia RJ. Long-term consequences from bladder perforation and/or violation in the presence of transitional cell carcinoma: results of a small series and a review of the literature. J Urol 1999;161:1128-32.

[28] Humphrey PA, Moch H, Cubilla AL, Ulbright TM, Reuter VE. The 2016 WHO classification of tumours of the urinary system and male genital organs-part B: prostate and bladder tumours. Eur Urol 2016;70:106-19.

[29] Ficarra V, Dalpiaz O, Alrabi N, Novara G, Galfano A, Artibani W. Correlation between clinical and pathological staging in a series of radical cystectomies for bladder carcinoma. BJU Int 2005;95:786-90.

[30] Kim JK, Park SY, Ahn HJ, Kim CS, Cho KS. Bladder cancer: analysis of multi-detector row helical CT enhancement pattern and accuracy in tumor detection and perivesical staging. Radiology 2004;231:725-31.

[31] Paner GP, Montironi R, Amin MB. Challenges in pathologic staging of bladder cancer: proposals for fresh approaches of assessing pathologic stage in light of recent studies and observations pertaining to bladder histoanatomic variances. Adv Anat Pathol 2017;24:113-27.

[32] Tritschler S, Mosler C, Straub J, et al. Staging of muscle-invasive bladder cancer: can computerized tomography help us to decide on local treatment? World J Urol 2012;30:827-31.

[33] Woo S, Suh CH, Kim SY, Cho JY, Kim SH. Diagnostic performance of MRI for prediction of muscle-invasiveness of bladder cancer: a systematic review and meta-analysis. Eur J Radiol 2017;95:46-55.

[34] Huang L, Kong Q, Liu Z, Wang J, Kang Z, Zhu Y. The Diagnostic value of MR imaging in differentiating T staging of bladder cancer: a meta-analysis. Radiology 2018;286:502-11.

[35] Kim B, Semelka RC, Ascher SM, Chalpin DB, Carroll PR, Hricak H. Bladder tumor staging: comparison of contrast-enhanced CT, T1- and T2weighted MR imaging, dynamic gadolinium-enhanced imaging, and late gadolinium-enhanced imaging. Radiology 1994;193:239-45.

[36] Johnson W, Taylor MB, Carrington BM, Bonington SC, Swindell R. The value of hyoscine butylbromide in pelvic MRI. Clin Radiol 2007;62:1087-93.

[37] Barentsz JO, Ruijs SH, Strijk SP. The role of MR imaging in carcinoma of the urinary bladder. AJR Am J Roentgenol 1993;160:937-47.

[38] Panebianco V, Barchetti F, de Haas RJ, et al. Improving staging in bladder cancer: the increasing role of multiparametric magnetic resonance imaging. Eur Urol Focus 2016;2:113-21.

[39] Takeuchi M, Sasaki S, Ito M, et al. Urinary bladder cancer: diffusionweighted MR imaging-accuracy for diagnosing $\mathrm{T}$ stage and estimating histologic grade. Radiology 2009;251:112-21.

[40] Takeuchi M, Sasaki S, Naiki T, et al. MR imaging of urinary bladder cancer for T-staging: a review and a pictorial essay of diffusionweighted imaging. J Magn Reson Imaging 2013;38:1299-309.

[41] Donaldson SB, Bonington SC, Kershaw LE, et al. Dynamic contrastenhanced MRI in patients with muscle-invasive transitional cell carcinoma of the bladder can distinguish between residual tumour and post-chemotherapy effect. Eur J Radiol 2013;82:2161-8.

[42] Narumi Y, Kadota T, Inoue E, et al. Bladder tumors: staging with gadolinium-enhanced oblique MR imaging. Radiology 1993;187:145-50.

[43] Hayashi N, Tochigi H, Shiraishi T, Takeda K, Kawamura J. A new staging criterion for bladder carcinoma using gadolinium-enhanced magnetic resonance imaging with an endorectal surface coil: a comparison with ultrasonography. BJU Int 2000;85:32-6.

[44] Zhou G, Chen X, Zhang J, Zhu J, Zong G, Wang Z. Contrast-enhanced dynamic and diffusion-weighted MR imaging at 3.0T to assess aggressiveness of bladder cancer. Eur J Radiol 2014;83:2013-8.

[45] Naish JH, McGrath DM, Bains LJ, et al. Comparison of dynamic contrast-enhanced MRI and dynamic contrast-enhanced CT biomarkers in bladder cancer. Magn Reson Med 2011;66:219-26.

[46] Panebianco V, De Berardinis E, Barchetti G, et al. An evaluation of morphological and functional multi-parametric MRI sequences in classifying non-muscle and muscle invasive bladder cancer. Eur Radiol 2017;27:3759-66.

[47] Cheong JJW, Woodward PJ, Maria A, Davis CJ. From the archives of the AFIP inflammatory and nonneoplastic bladder masses: radiologic-pathologic objectives. RadioGraphics 2006;1595:1847-69. 
[48] Ma W, Kang SK, Hricak H, Gerst SR, Zhang J. Imaging appearance of granulomatous disease after intravesical Bacille Calmette-Guerin (BCG) treatment of bladder carcinoma. AJR Am J Roentgenol 2009; 192:1494-500

[49] El-Assmy A, Abou-El-Ghar ME, Refaie HF, Mosbah A, El-Diasty T. Diffusion-weighted magnetic resonance imaging in follow-up of superficial urinary bladder carcinoma after transurethral resection: initial experience. BJU Int 2012;110:E622-7.

[50] Wang HJ, Pui MH, Guo Y, Yang D, Pan BT, Zhou XH. Diffusionweighted MRI in bladder carcinoma: the differentiation between tumor recurrence and benign changes after resection. Abdom Imaging 2014;39:135-41.

[51] Narumi Y, Kadota T, Inoue E, et al. Bladder wall morphology: in vitro MR imaging-histopathologic correlation. Radiology 1993; 187:151-5.

[52] Xiao GQ Rashid H. Bladder neck urothelial carcinoma: a urinary bladder subsite carcinoma with distinct clinicopathology. Int J Surg Pathol 2015;23:517-23.

[53] Wang HJ, Pui MH, Guan J, et al. Comparison of early submucosal enhancement and tumor stalk in staging bladder urothelial carcinoma. AJR Am J Roentgenol 2016;207:797-803.

[54] Kulkarni GS, Alibhai SM, Finelli A, et al. Cost-effectiveness analysis of immediate radical cystectomy versus intravesical Bacillus Calmette-Guerin therapy for high-risk, high-grade (T1G3) bladder cancer. Cancer 2009;115:5450-9.

[55] Lammle M, Beer A, Settles M, Hannig C, Schwaibold H, Drews C. Reliability of MR imaging-based virtual cystoscopy in the diagnosis of cancer of the urinary bladder. AJR Am J Roentgenol 2002; 178:1483-8.

[56] Gore JL, Lai J, Setodji CM, Litwin MS, Saigal CS. Urologic Diseases in America Project. Mortality increases when radical cystectomy is delayed more than 12 weeks: results from a Surveillance, Epidemiology, and End Results-Medicare analysis. Cancer 2009;115:988-96.

[57] van den Bosch S, Alfred Witjes J. Long-term cancer-specific survival in patients with high-risk, non-muscle-invasive bladder cancer and tumour progression: a systematic review. Eur Urol 2011;60:493500.

[58] Oughton JB, Poad H, Twiddy M, et al. Radical cystectomy (bladder removal) against intravesical BCG immunotherapy for high-risk non-muscle invasive bladder cancer (BRAVO): a protocol for a randomised controlled feasibility study. BMJ Open 2017;7:e017913.

[59] Wang F, Wu LM, Hua XL, Zhao ZZ, Chen XX, Xu JR. Intravoxel incoherent motion diffusion-weighted imaging in assessing bladder cancer invasiveness and cell proliferation. J Magn Reson Imaging 2018;47:1054-60.

[60] Thomas F, Noon AP, Rubin N, Goepel JR, Catto JW. Comparative outcomes of primary, recurrent, and progressive high-risk nonmuscle-invasive bladder cancer. Eur Urol 2013;63:145-54.

[61] International Collaboration of Trialists, Medical Research Council Advanced Bladder Cancer Working Party (now the National Cancer Research Institute Bladder Cancer Clinical Studies Group), European Organisation for Research and Treatment of Cancer Genito-urinary Tract Cancer Group, et al. International phase III trial assessing neoadjuvant cisplatin, methotrexate, and vinblastine chemotherapy for muscle-invasive bladder cancer: long-term results of the BA06 30894 trial. J Clin Oncol 2011;29:2171-7.

[62] Seisen T, Sun M, Lipsitz SR, et al. Comparative effectiveness of trimodal therapy versus radical cystectomy for localized muscleinvasive urothelial carcinoma of the bladder. Eur Urol 2017;72:483-7.

[63] Schrier BP, Peters M, Barentsz JO, Witjes JA. Evaluation of chemotherapy with magnetic resonance imaging in patients with regionally metastatic or unresectable bladder cancer. Eur Urol 2006;49:698-703.
[64] Barentsz JO, Berger-Hartog O, Witjes JA, et al. Evaluation of chemotherapy in advanced urinary bladder cancer with fast dynamic contrast-enhanced MR imaging. Radiology 1998;207:791-7.

[65] Nguyen HT, Jia G, Shah ZK, et al. Prediction of chemotherapeutic response in bladder cancer using $\mathrm{K}$-means clustering of dynamic contrast-enhanced (DCE)-MRI pharmacokinetic parameters. J Magn Reson Imaging 2015;41:1374-82.

[66] Eisenhauer EA, Therasse P, Bogaerts J, et al. New response evaluation criteria in solid tumours: revised RECIST guideline (version 1.1). Eur J Cancer 2009;45:228-47.

[67] Yoshida S, Koga F, Kawakami S, et al. Initial experience of diffusionweighted magnetic resonance imaging to assess therapeutic response to induction chemoradiotherapy against muscle-invasive bladder cancer. Urology 2010;75:387-91.

[68] Johnson RJ, Carrington BM, Jenkins JP, Barnard RJ, Read G, Isherwood I. Accuracy in staging carcinoma of the bladder by magnetic resonance imaging. Clin Radiol 1990;41:258-63.

[69] Barentsz JO, Jager GJ, van Vierzen PB, et al. Staging urinary bladder cancer after transurethral biopsy: value of fast dynamic contrastenhanced MR imaging. Radiology 1996;201:185-93.

[70] Splinter TA, Scher HI, Denis L, et al. The prognostic value of the pathological response to combination chemotherapy before cystectomy in patients with invasive bladder cancer. European Organization for Research on Treatment of Cancer-Genitourinary Group. J Urol 1992;147:606-8.

[71] Grossman HB, Natale RB, Tangen CM, et al. Neoadjuvant chemotherapy plus cystectomy compared with cystectomy alone for locally advanced bladder cancer. N Engl J Med 2003;349:859-66.

[72] Sternberg CN, Pansadoro V, Calabro F, et al. Can patient selection for bladder preservation be based on response to chemotherapy? Cancer 2003;97:1644-52.

[73] Mak RH, Hunt D, Shipley WU, et al. Long-term outcomes in patients with muscle-invasive bladder cancer after selective bladder-preserving combined-modality therapy: a pooled analysis of radiation therapy oncology group protocols 8802, 8903, 9506, 9706, 9906, and 0233. J Clin Oncol 2014;32:3801-9.

[74] Hafeez S, Horwich A, Omar O, et al. Selective organ preservation with neo-adjuvant chemotherapy for the treatment of muscle invasive transitional cell carcinoma of the bladder. $\mathrm{Br} \mathrm{J}$ Cancer 2015;112:1626-35.

[75] Efstathiou JA, Spiegel DY, Shipley WU, et al. Long-term outcomes of selective bladder preservation by combined-modality therapy for invasive bladder cancer: the MGH experience. Eur Urol 2012;61:705-11.

[76] Rosenblatt R, Sherif A, Rintala E, et al. Pathologic downstaging is a surrogate marker for efficacy and increased survival following neoadjuvant chemotherapy and radical cystectomy for muscleinvasive urothelial bladder cancer. Eur Urol 2012;61:1229-38.

[77] Yoshida S, Koga F, Kobayashi S, et al. Role of diffusion-weighted magnetic resonance imaging in predicting sensitivity to chemoradiotherapy in muscle-invasive bladder cancer. Int J Radiat Oncol Biol Phys 2012;83:e21-7.

[78] Sevcenco S, Haitel A, Ponhold L, et al. Quantitative apparent diffusion coefficient measurements obtained by 3-Tesla MRI are correlated with biomarkers of bladder cancer proliferative activity. PLoS One 2014;9:e106866.

[79] Kobayashi S, Koga F, Kajino K, et al. Apparent diffusion coefficient value reflects invasive and proliferative potential of bladder cancer. J Magn Reson Imaging 2014;39:172-8.

[80] Alfred Witjes J, Lebret T, Comperat EM, et al. Updated 2016 EAU guidelines on muscle-invasive and metastatic bladder cancer. Eur Urol 2017;71:462-75.

[81] Advanced Bladder Cancer (ABC) Meta-analysis Collaboration. Neoadjuvant chemotherapy in invasive bladder cancer: update of 
a systematic review and meta-analysis of individual patient data. Eur Urol 2005;48:202-5, discussion 5-6.

[82] Hafeez S, Koh MD, Sohaib A, Huddart R. Use of diffusion weightedMRI (DW-MRI) as a prognostic biomarker of survival and time to cystectomy in muscle invasive bladder cancer (MIBC) following organ conserving treatment. Eur J Cancer 2017;72:S192.

[83] Hafeez S, Koh M, Sohaib A, Huddart R. PD-0464: assessing response to chemotherapy with diffusion weighted MRI (DW-MRI) in muscle invasive bladder cancer (MIBC). Radiother Oncol 2013;106(Suppl 2):S180-1.
[84] Nguyen HT, Mortazavi A, Pohar KS, et al. Quantitative assessment of heterogeneity in bladder tumor MRI diffusivity: can response be predicted prior to neoadjuvant chemotherapy? Bladder Cancer 2017;3:237-44

[85] O'Connor JP, Aboagye EO, Adams JE, et al. Imaging biomarker roadmap for cancer studies. Nat Rev Clin Oncol 2017;14:169-86.

[86] Weinreb JC, Barentsz JO, Choyke PL, et al. PI-RADS Prostate Imaging-Reporting and Data System: 2015, version 2. Eur Urol 2016;69:16-40.

\section{www.esukidneytransplant.org}

\section{Ist ESU-ESTU Masterclass on Kidney transplant}

15-16 November 2018, Madrid, Spain

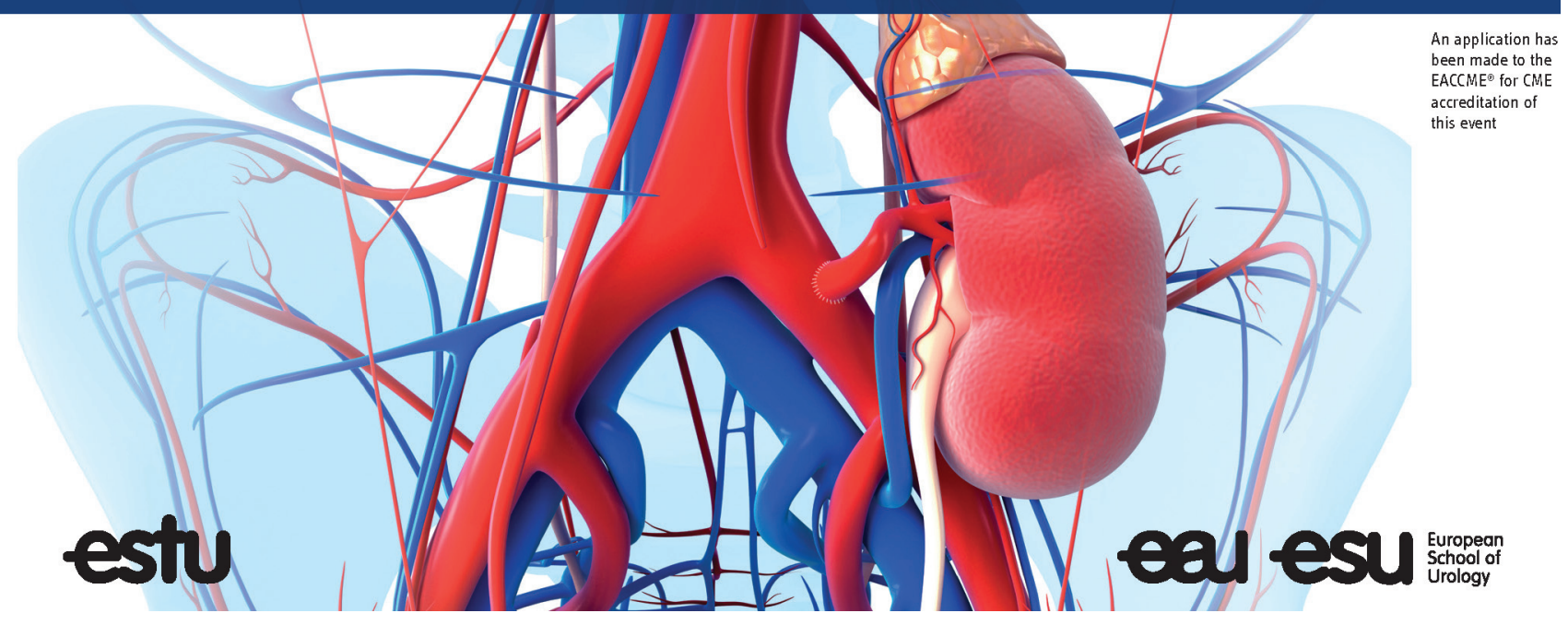

